\title{
Pseudogenes act as microRNA decoys
}

A recent study revises our view of pseudogenes as non-functional relics of evolution by showing that transcripts produced from pseudogenes (competitive endogenous RNAs (ceRNAs)) regulate the effects of microRNAs (miRNAs) on their targets by competing for miRNA binding.

Poliseno, Salmena et al. investigated $P$ TENP 1 , a pseudogene of the tumour suppressor gene PTEN. They

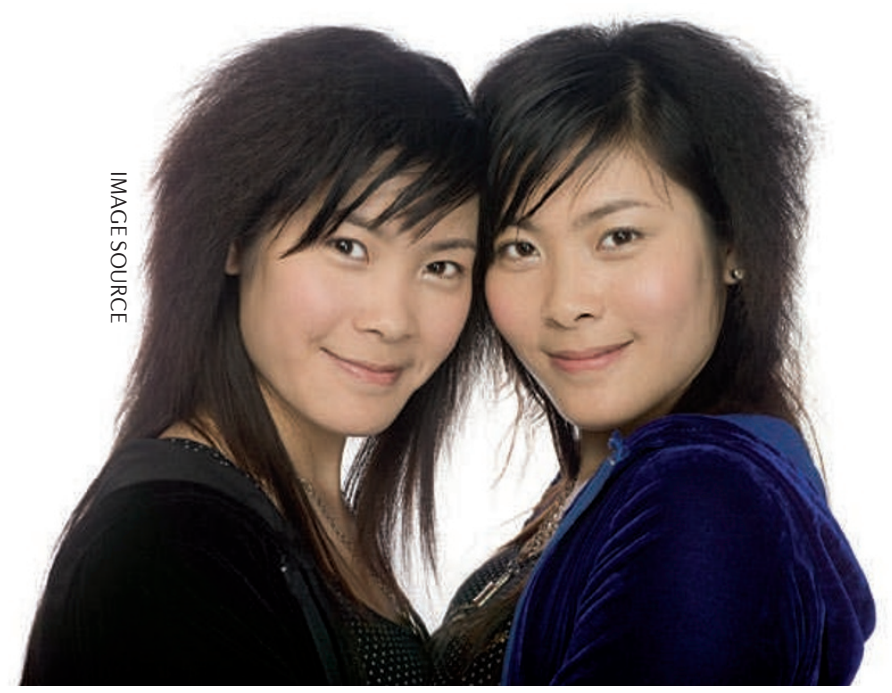

found several binding sites for miRNAs that target PTEN in the 3' UTR of PTENP1 mRNA. Furthermore, in prostate cancer cells, expression of PTEN-targeting miRNAs led to downregulation of both PTEN and PTENP1 mRNAs, confirming that $P T E N P 1$ is subject to miRNA-mediated regulation.

Does miRNA binding by PTENP1 affect the tumour suppressive activities of PTEN? Overexpression of the PTENP1 3' UTR led to increased levels of PTEN transcripts and protein and to growth inhibition in prostate cancer cells. This de-repression of PTEN by the PTENP1 3' UTR was abrogated in DICER-null colon carcinoma cells, which are defective in miRNA processing. These findings suggest that mature miRNAs are needed for PTENP1 to regulate PTEN levels.

The authors also identified focal copy number losses at the PTENP1 locus associated with downregulation of PTEN expression in samples from sporadic colon cancer patients.

They suggest that PTENP1 could be considered a tumour suppressor gene and that there might be selection for loss of PTENP1 during tumorigenesis.

How widespread is this mechanism of regulating cancer genes? The authors found miRNA-binding sites in pseudogenes of other cancerrelated genes. They also demonstrated a similar relationship between the oncogene KRAS and its pseudogene KRAS1P to that between PTEN and PTENP1. Because each miRNA has multiple targets and because genes can often have several related pseudogenes, complex networks of regulation may exist. This decoy mechanism may not be limited to pseudogene transcripts, so the next steps will be to investigate whether other non-coding RNAs can act as trans-regulators by miRNA binding and how generally this mechanism applies outside of cancer genes. The insights from this study may also help to identify new therapeutic targets in cancer.

Meera Swami

ORIGINAL RESEARCH PAPER Poliseno, L. et al. A coding-independent function of gene and pseudogene mRNAs regulates tumour biology. Nature 465, 1033-1038 (2010) 